\title{
PSIDIUM GUAJAVA: A NOVEL PLANT IN THE SYNTHESIS OF SILVER NANOPARTICLES FOR BIOMEDICAL APPLICATIONS
}

\author{
SHARMILA $\mathbf{C}^{1}$, RANJITH KUMAR R ${ }^{2}$, CHANDAR SHEKAR $\mathbf{B}^{3 *}$ \\ ${ }^{1}$ Department of Physics, PSGR Krishnammal College for Women, Coimbatore - 641 004, Tamil Nadu, India. ${ }^{2}$ Department of Biotechnology, \\ Nehru Arts and Science College, Coimbatore - 641 105, Tamilnadu, India. ${ }^{3}$ Department of Physics, Kongunadu Arts and Science College, \\ Coimbatore - 641 029, Tamil Nadu, India. Email: chandar.bellan@gmail.com
}

Received: 14 August 2017, Revised and Accepted: 27 October 2017

ABSTRACT

Objective: Synthesis of silver nanoparticles (AgNPs) using a simple, cost-effective and environmentally friendly green route approach and to study the antibacterial activity of AgNPs against human pathogens.

Methods: Green route approach is used to synthesize AgNPs using Psidium guajava leaf extract. Fourier transform infrared (FTIR) was used to identify the presence of the functional group. X-ray diffraction (XRD) was used to analyze the structure of prepared AgNPs. Energy dispersive X-ray was used to the characteristic to the composition of the prepared nanoparticles. Size and morphology of the prepared AgNPs were investigated using field emission scanning electron microscopy (FESEM) and transmission electron microscopy (TEM) analysis. Antibacterials efficiency of prepared AgNPs was tested against Escherichia coli and Staphylococcus aureus by well diffusion methods.

Results: FTIR study shows the presence of different functional groups present in the leaves mediated AgNPs. The XRD studies yield diffraction peaks corresponding to face-centered cubic structure of Ag crystals. Spherical shaped AgNPs with a particle size of about $\sim 55 \mathrm{~nm}$ were evidenced using FESEM and TEM analysis. Energy dispersive spectrum of the synthesized AgNPs confirms the presence of silver in the prepared nanoparticles. From UV-VIS analysis it is shown that the absorption band was red-shifted from $430 \mathrm{~nm}$ to $456 \mathrm{~nm}$. The prepared AgNPs shows good antibacterial activity against E. coli and S. aureus.

Conclusions: P. guajava leaf extract is a potential reducing agent to synthesize AgNPs. The green synthesis approach provides cost-effective and ecofriendly nanoparticles, which could be used in biomedical applications.

Keywords: Psidium guajava, Silver nanoparticles, Fourier transform infrared, Transmission electron microscopy, Antibacterial activity

(C) 2018 The Authors. Published by Innovare Academic Sciences Pvt Ltd. This is an open access article under the CC BY license (http://creativecommons. org/licenses/by/4. 0/) DOI: http://dx.doi.org/10.22159/ajpcr.2018.v11i1.21999

\section{INTRODUCTION}

Nanomaterials research has received the considerable attention of researchers worldwide due to its unique properties [1,2]. Nanomaterials are expected to have a wide range of applications in various fields such as electronics, optical communications, and biological system $[3,4]$. These applications are based on factors such as their physical property, huge surface area, and small size which offer the possibility for manipulation and room for accommodating multiple functionalities. The very promising and rapidly growing field of nanotechnology is in medicine and sustainable energy resources [5]. Silver nanoparticles (AgNPs) are widely being used because it is more effective against bacteria's. In the synthesis of AgNPs, the processing condition need to be controlled in such a manner that the resulting nanoparticles have an identical shape, identical size, and individually dispersed with no agglomeration [6,7]. AgNPs are mostly preferred for biomedical applications because; $\mathrm{Ag}^{+}$ions are highly compatible for the human body. Many methods have been reported for the synthesis of AgNPs [8-11], but only a few methods have produced a size controlled and monodispersed AgNPs. These monodispersed AgNPs have a great effect on their physical and chemical properties. All the methods have concentrated on preparing particles of very small size and definite shape which focuses for different applications [12-14].

The size of the metallic collides varies significantly with the type of reducing agent. To optimize AgNPs, the reducing agent was selected from plant extract by green process. Country like India has a rich source of medicinal plants and plant products. From centuries to till date the medicinal plants have been extensively used in Ayurveda. Among these medicinal plants, Psidium guajava (Guava) plant has showed high medicinal value. Studies have shown that AgNPs prepared from guava leaves were used in different application [15]. In most ethnopharmacological studies of $P$. guajava leaves showed that the leaf extract has antidiarrheal, antibacterial, anti-inflammatory, and anticancer activities [16-18]. P. guajava commonly called as guava plant has been studied for many years because the plant had interesting biological and pharmacological activities. The plant also has a potential antioxidant activity against free radical scavenging [19]. Hence, $P$. guajava leaf extract was used as a reducing agent in the preparation of AgNPs. Previous investigations on P. guajava plant extract have led to the isolation of different classes of bioactive constituents such as triterpenoids, flavonoids, tannins, and carotenoids [20-23]. These organic molecules are responsible for the reduction, stabilization and capping of the resulting nanoparticles [24].

Many researchers have suggested that the reduction of metal nanoparticles was mainly due to plant phytochemical molecules and which is responsible for the size and shape of nanoparticles [24,25]. P. guajava leaf extract showed many phytochemical molecules and its hold high amount of novel caryophyllene based terpenoid (Guajadial-1) compound and its response for the various biomedical applications [21]. In this present work, an attempt has been made to prepare AgNPs using P. guajava leaf extract and study the antibacterial effect of the prepared nanoparticles against human pathogens.

\section{METHODS}

Chemicals and reagents

Silver nitrate (99\% purity) was purchased from HiMedia, India Pvt. Ltd and the chemical used was of analytical grade purity. P. guajava leaves 
used in this experiment were collected from the local residence around Coimbatore, Tamil Nadu, India.

\section{Preparation of leaf extract}

Fresh leaves of P. guajava were collected and cut into small pieces. These leaves were washed thoroughly for several times with double distilled water to remove dust and other particles attached to the surface of leaves. P. guajava leaf extract was prepared by weighing $20 \mathrm{~g}$ of freshly cut leaves with $200 \mathrm{ml}$ of double distilled water boiled at $60^{\circ} \mathrm{C}$ in Erlenmeyer flask for $20 \mathrm{~min}$. The P. guajava leaf extract was then filtered through Whatman No.1 filter paper [26]. The filtered was used for the synthesis of nanoparticles in room temperature.

\section{Green synthesis of AgNPs}

AgNPs were synthesized by mixing $100 \mathrm{ml}$ of $1 \times 10^{-3} \mathrm{M}$, aqueous silver nitrate with $5 \mathrm{ml}$ of $P$. guajava leaf extract and are stirred vigorously for $20 \mathrm{~min}$ in a magnetic stirrer at room temperature. It was observed that color of the reaction mixture changed from light green to reddish brown that indicating the formation of AgNPs [27].

\section{Characterizations of AgNPs}

The Fourier transform infrared (FTIR) spectra for $P$. guajava leaf extract, and AgNPs were obtained in range $4000-500 / \mathrm{cm}$ with IR-Affinity-1, Shimadzu, and FTIR Spectrometer. X-ray diffraction (XRD) (Shimadzu Lab, XRD 6000, Japan) was analyzed for the range of $10^{\circ}-80^{\circ}$ by powder XRD using $\mathrm{CuK} \alpha$ radiation (1.5406 $\mathrm{A}$ ). Full width half maximum (FWHM) ( $\beta$ ) values and diffraction angles $(\theta)$ are used to calculate the crystallite size and peak boarding of prepared AgNPs. The surface morphology of the P. guajava leaf extract mediated AgNPs was carried out using JEOL-JSM-6490LA field emission scanning electron microscopy (FESEM) (JEOL, Tokyo, Japan) operated at $20 \mathrm{kV}$ with a counting rate of $2838 \mathrm{cps}$. The composition of the prepared AgNPs was studied by energy dispersive X-ray (EDAX) spectroscopy (JEOL, JSM 6390, Japan). The atomic and weight percentage of the existing elements were calculated using EDAX spectrum. The optical properties of prepared AgNPs were characterized by UV-Visible Spectroscopy (CYBERLAB, UV-100) double beam spectrophotometer.

\section{The antibacterial activity}

The antibacterial activity of prepared AgNPs was investigated by a standard zone of inhibition microbiology assay against Gram-positive (Staphylococcus aureus) and Gram-negative (Escherichia coli) human pathogens. The clinically isolated Gram-negative bacteria E. coli and Gram-positive $S$. aureus were obtained from the clinical testing lab, Coimbatore, Tamil Nadu, India. Muller Hinton Agar plate was used to swab pathogenic bacteria culture (S. aureus and E. coli) from fresh culture $\left(1.5 \times 10^{8} \mathrm{CFU} / \mathrm{mL}\right)$ by sterile cotton scrub. The surface of the medium was allowed to dry for about $3 \mathrm{~min}$. The wells $(6 \mathrm{~mm})$ were punched over the agar plates using sterile gel puncher. Different concentrations $(10,25$, and $50 \mu \mathrm{l})$ of prepared AgNPs were loaded into wells on test plates. The plates were incubated for $24 \mathrm{~h}$ at $37^{\circ} \mathrm{C}$, and the zone of inhibition was measured in mille meter. The antibacterial efficiency of prepared AgNPs was compared with standard drug ofloxacin disc $(5 \mu \mathrm{g} / \mathrm{disc})$

\section{RESULTS AND DISCUSSION}

The AgNPs which are less likely to cause ecological effect have been identified as a potential replacement of synthetic chemical insecticides; hence, the need to use green synthesized AgNPs for the control of disease vectors. P. guajava leaf extract showed different classes of bioactive constituents, which is responsible for the biological applications. Previous researcher reported that the $P$. guajava leaf extract showed terpenoids compounds in high concentration $(>50 \%)$ when compared with other plant phytochemicals such as flavonoids, tannins, etc. [15,20]. Guajadial (1) is a novel caryophyllene based meroterpenoid, was isolated from the leaves of P. guajava [21], which may be responsible for the reduction of AgNPs. The possible reduction of Ag from Guajadial (1) is shown in Fig. 1.

\section{FTIR spectroscopy}

To support the possible pathway of green synthesized AgNPs the FTIR analysis for the guava leaf extract and the synthesized AgNPs were carried out. Fig. 2 shows the FTIR spectrum of the P. guajava leaf extract and the synthesized AgNPs.

FTIR peaks at $3373(-\mathrm{OH}), 2788(\mathrm{CH}-0), 1645(\mathrm{C}=0), 1451$ (C-C=C), 1363 (N-0), 1023 (C-0), 825 (alkenes), and 702 (aromatic rings), represents the different functional groups present in the $P$. guajava leaf extract. FTIR peaks at 1529 (C-C), 1341 (N-O), 962 (C-C), and 716 (R-CH) represents the different functional groups present in AgNPs after reduction. From the FTIR spectrum it is clearly seen that the AgNPs have been accommodated into the $-\mathrm{OH}$ stretching and have been reduced into $\mathrm{O}^{-}$and $\mathrm{Ag}^{+}$ions. The intensity of all the other peaks in the synthesized nanoparticles has been reduced and is shifted toward the lower wavelength.

\section{XRD analysis}

The XRD peaks of the green route synthesized AgNPs are shown in Fig. 3. The XRD spectrum shows four main predominant Braggs diffraction peaks which are positioned at $2 \theta$ values of $38.0^{\circ}, 44.1^{\circ}, 64.4^{\circ}$, and $77.4^{\circ}$ which corresponds to $111,200,220$, and 311 facets of the face-centered cubic structure of AgNPs. These diffraction peaks are consistent with the standard Ag crystals (JCPDS Card No.4-0783), indicating that the synthesized AgNPs are of crystalline in nature. These results are in good correlation with previously reported results of AgNPs [28]. The unassigned peaks $\left(^{*}\right)$ observed in the XRD spectrum indicates that the crystallization of plant phytochemicals phase presence in P. guajava leaf extract occurred on the surface of the AgNPs.

The crystallite size of the green synthesis AgNPs was calculated by the Debye-Scherrer equation, $\mathrm{D}=\mathrm{K} \lambda / \beta \cos \theta$. Where $\mathrm{D}=$ average crystallite size, $\mathrm{K}$ - shape factor $(0.9), \lambda$ - wavelength of $\mathrm{Cu} \mathrm{K} \alpha$ radiation (1.541 $\AA$ ), $\beta$ - FWHM of refection (in radians) located at $2 \theta$ and $\theta$ - angle of reflection (in degrees) was used to relate the crystallite size to the line broadening. The average crystallite size of prepared AgNPs was found to be about $25.8 \mathrm{~nm}$.

FESEM and transmission electron microscopy (TEM) analysis FESEM micrographs of AgNPs synthesized using P. guajava plant extract shows that the AgNPs are spherical in shape with average grain size

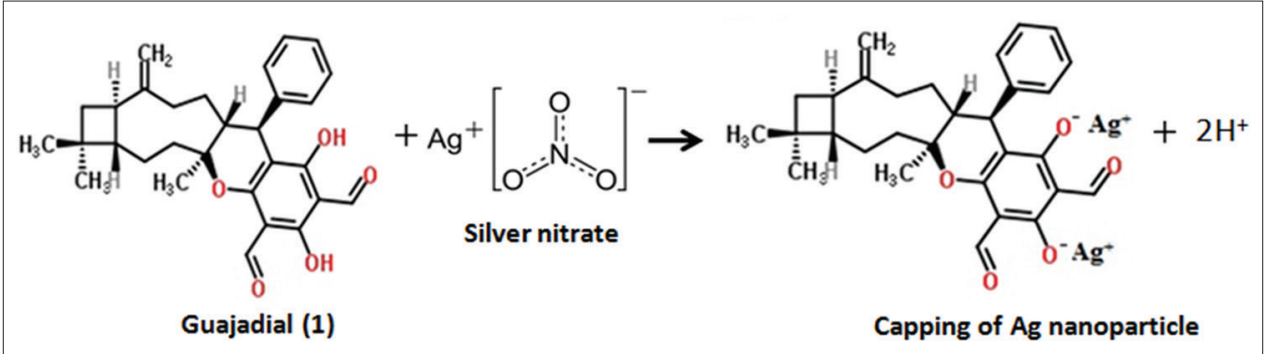

Fig. 1: Possible reduction of silver nanoparticles from Guajadial (1) 


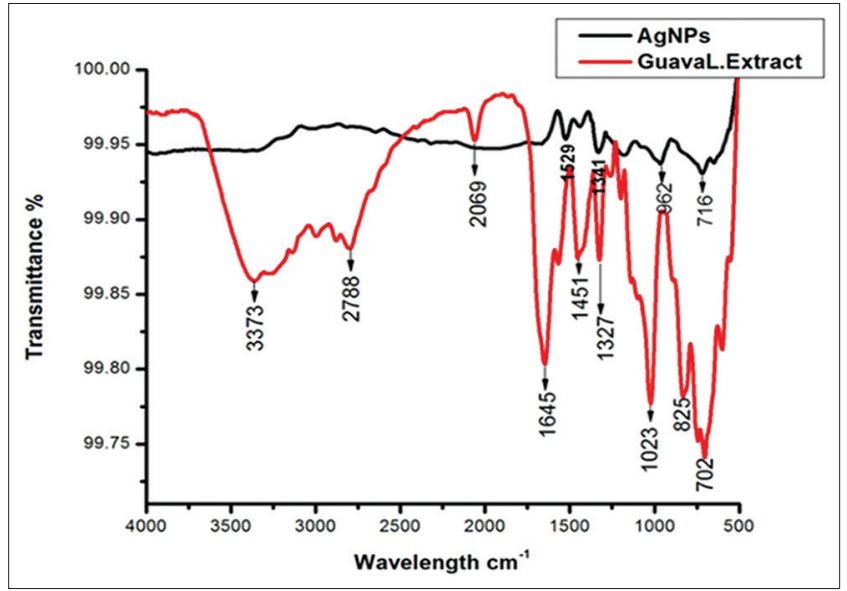

Fig. 2: Fourier transform infrared spectrum of silver nanoparticles and Psidium guajava leaf extract

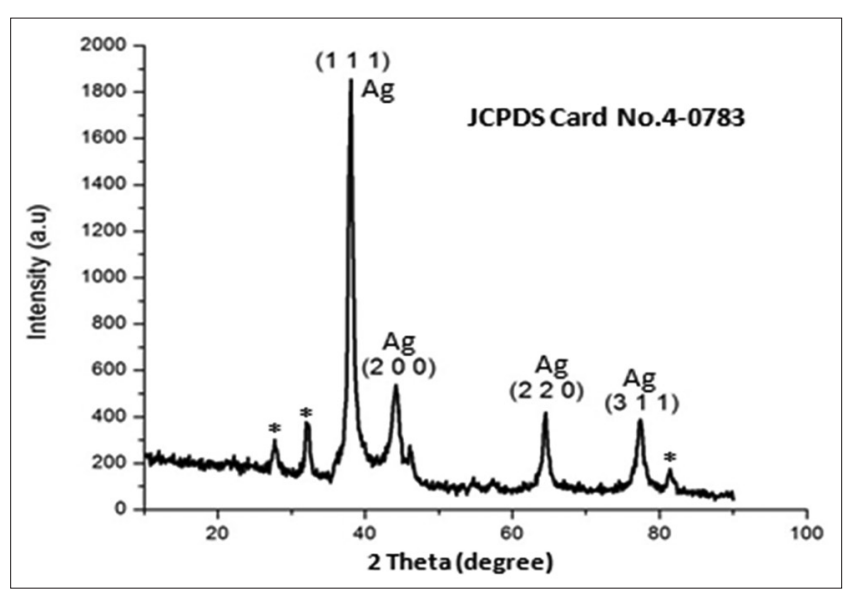

Fig. 3: X-ray diffraction spectrum silver nanoparticles

of $\sim 80 \mathrm{~nm}$. The FESEM micrographs of different magnifications are shown in Fig. 4.

TEM image of green synthesized AgNPs shown in Fig. 5. The micrograph technique shows that the resultant nanoparticles are very fine and well dispersed. The nanoparticles obtained are all spherical in shape with an average size of $55 \mathrm{~nm}$. Constituents present in P. guajava leaves were capped in the AgNPs, and these constituents might have prevented the nanoparticles from agglomeration.

The energy dispersive spectrum (EDS) was done along with the FESEM analysis. The EDS spectra of the green route prepared AgNPs are shown in Fig. 6. The spectrum reveals the presence of silver at $\sim 3 \mathrm{keV}$. In general, elemental silver gives a signal in this energy region $[29,30]$. The EDS composition analysis confirmed the presence of Ag (93\% wt) in the prepared AgNPs.

\section{UV-visible spectroscopy}

Absorption spectral analysis is the most commonly used technique in the identification of nanoparticles formation. Reduction of silver ions into AgNPs using P. guajava leaf extract was evidenced visually by a change in color from light yellow to dark brown [31]. Color intensity increases with increase in incubation time. Initially, when adding $100 \mathrm{ml}$ of $1 \times 10^{-3} \mathrm{M}$ aqueous silver nitrate with $5 \mathrm{ml}$ of leaf extract, the color of the solution was found to be light yellow after $15 \mathrm{~min}$ the change of color from light yellow to reddish brown was evidenced, and after 1 hour the color changed from reddish brown to dark brown, this effect may be due to surface plasmon vibration [32,33]. The color of the colloidal solution of AgNPs depends on the size and shape of the

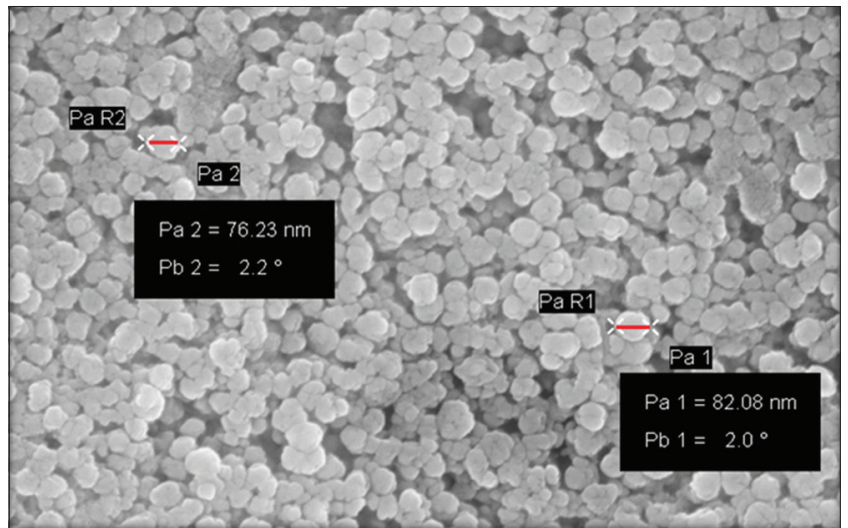

Fig. 4: Field emission scanning electron microscopy images of green synthesized silver nanoparticles

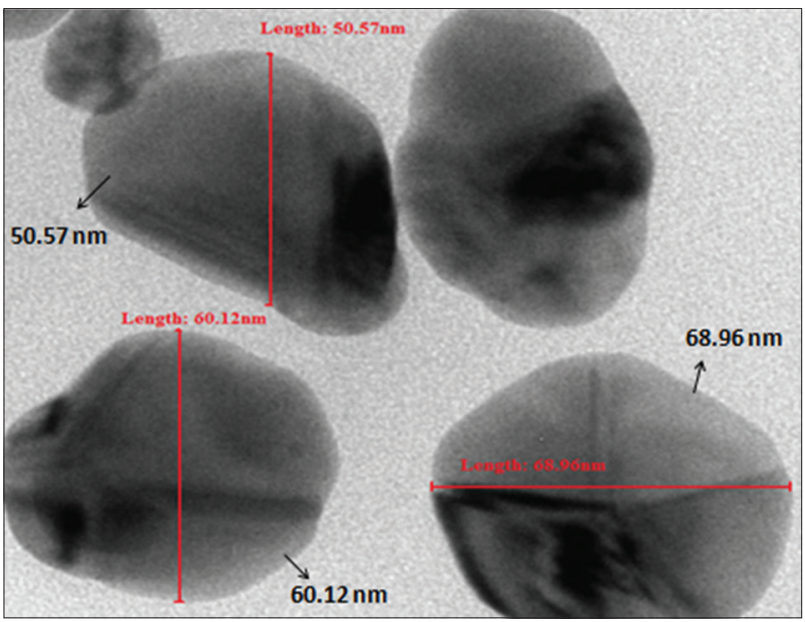

Fig. 5: Transmission electron microscopy image for the green synthesized silver nanoparticles

nanoparticles. Usually, metal nanoparticles were analyzed in the range from 300 to $800 \mathrm{~nm}$ wavelength, and formation of AgNPs can be detected commonly from the absorption measurement in the wavelength ranges of 400-450 nm [34]. In this present study, the absorption band was observed from 430 to $456 \mathrm{~nm}$ for different incubation time (Fig. 7). The intensity of the absorption peak increases steadily as a function of time and was highest for $1 \mathrm{~h}$. The UV-spectra recorded after $1 \mathrm{~h}$ does not show any increase in the intensity of absorption spectrum, from which it is confirmed that the reaction was completed within $1 \mathrm{~h}$.

\section{Antibacterial activity of AgNPs}

The agar well diffusion method was employed for screening the antibacterial activity of AgNPs against Gram-positive and Gramnegative bacteria. The green route synthesized AgNPs showed antibacterial activity in all concentrations $(10,25$, and $50 \mu \mathrm{L})$ against both test pathogens such as S. aureus and E. coli and also it indicated the dose depended on mechanisms. The antibacterial activity of AgNPs was increasing with the increasing the concentration of AgNPs against test pathogens. The prepared AgNPs showed a maximum zone of inhibition in all three concentration against $E$. coli compare to $S$. aureus (Fig. 8).

At the concentration of $50 \mu \mathrm{L}$, AgNPs solution shows a maximum zone of inhibition around $12 \mathrm{~mm}$ for both test pathogens. Whereas, $25 \mu \mathrm{L}$ showed a zone of inhibition around $11 \mathrm{~mm}$ for E. coli and $10 \mathrm{~mm}$ for S. aureus. The lowest concentration of $10 \mu \mathrm{L}$ AgNPs solution observed $9 \mathrm{~mm}$ for both tested human pathogens. The present study clearly indicates the antibacterial activity was dose-depended manner. The standard drug (ofloxacin) ( $5 \mu \mathrm{g} /$ disc) observed 19 and $12 \mathrm{~mm}$ against 


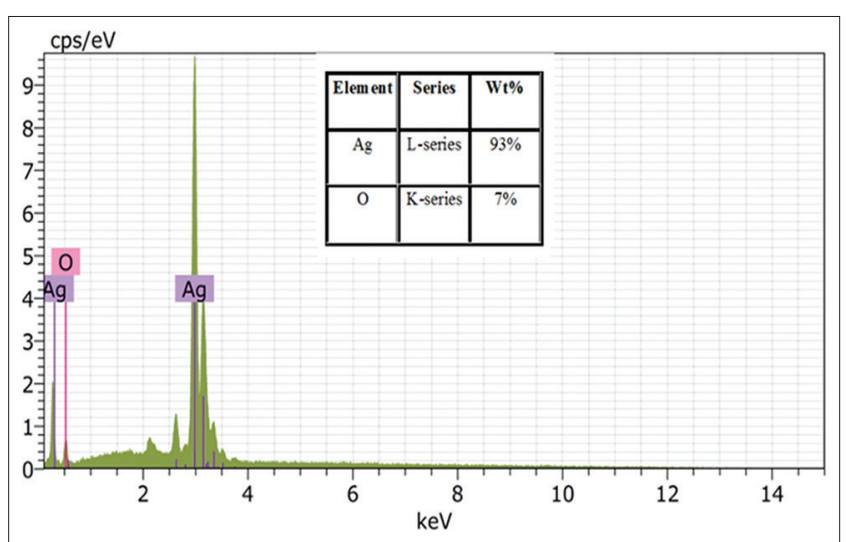

Fig. 6: Energy dispersive spectrum of silver nanoparticles

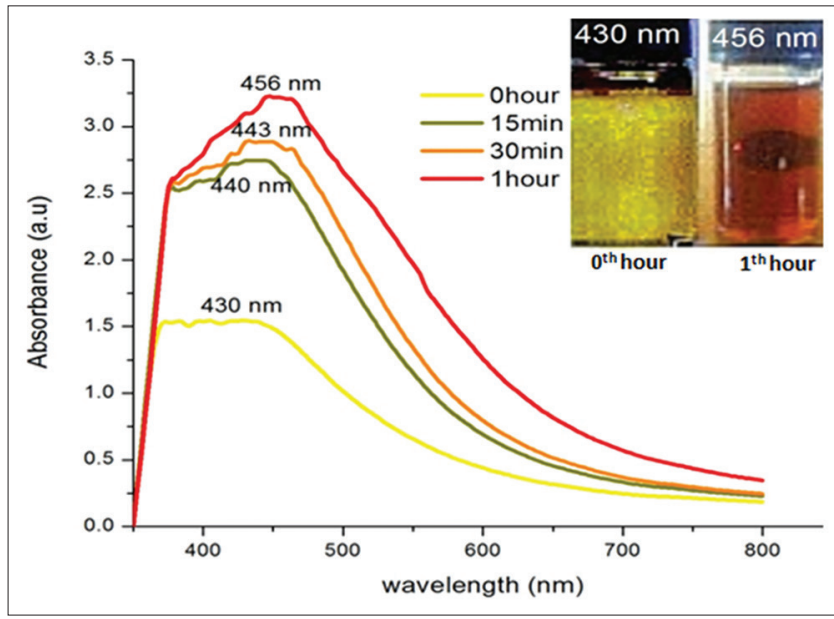

Fig. 7: UV-visible absorption spectra of silver nanoparticles for different time intervals

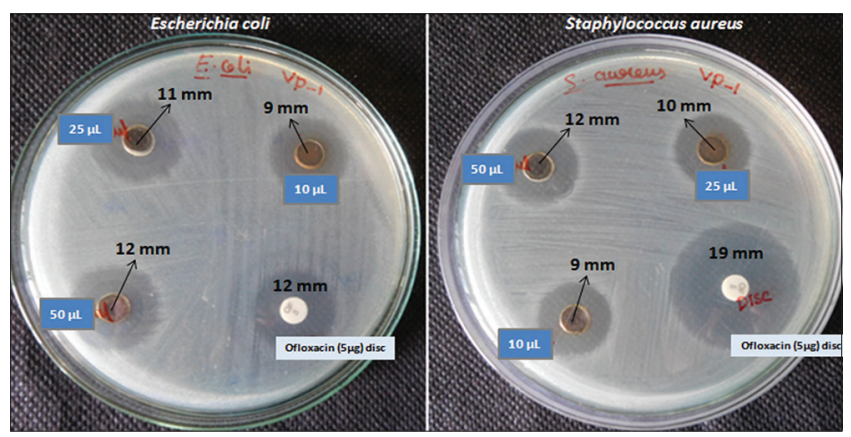

Fig. 8: Antibacterial activity of silver nanoparticles

S. aureus and E. coli. Nanoparticles may infiltrate the cells causing intracellular loss leading to cell death, and this inhibition depends on the concentration of the AgNPs [35,36]. The observed value of inhibition is in good agreement with recently reported values against E. coli and $S$. aureus using inorganic nanoparticles by our group [26,37]. In this present study reveals that the P. guajava leaf extract mediated green synthesized AgNPs are good antibacterial material against Gramnegative and Gram-negative pathogens.

\section{CONCLUSION}

The AgNPs were synthesized at room temperature using $P$. guajava leaf extract. The plant phytochemicals molecules are responsible for the reduction of AgNPs and enhanced antibacterial activity. FTIR analysis supports the presence of functional groups of biomolecules capped on the prepared AgNPs. These green routes synthesized AgNPs were preliminary identified by visual inspection where the change in color was evidenced, and then different characterization techniques were implemented to prove the presence of AgNPs. The absorption spectrum and XRD studies evidenced the presence of AgNPs. Electron microscope technique such as FESEM and TEM analysis confirmed the spherical shape and size of about $\sim 55 \mathrm{~nm}$ of the un-agglomerated AgNPs. The elemental analysis also confirms the presence of Ag in the prepared sample. The antibacterial activity of AgNPs was carried out against E. coli and S. aureus and its zone of inhibition was dose-dependent manner. Moreover, the present study demonstrated fast, eco-friendly, cost-effective and simply route to synthesize AgNPs from P. guajava leaf extract for biomedical applications.

\section{REFERENCES}

1. Senol AM, Metin O, Acar M, Onganer Y, Meral K. The interaction of fluorescent Pyronin Y molecules with monodisperse silver nanoparticles in chloroform. J Mol Struct 2016;1103:212-6.

2. Sathish S, Shekar BC, Nataraj D. Synthesis and characterization of indium aluminate $(\mathrm{InAlO}$ ) nanoparticles by wet chemical method. Adv Powder Tech 2014;25:1007-15

3. Darder M, Colilla M, Ruiz-Hitzky E. Citosan-clay nanocomposites: Applications as electrochemical sensors. App Clay Sci 2005;28:199-208.

4. Nair LS, Laurencin CT. Silver nanoparticles: Synthesis and therapeutic applications. J Biomed Nanotech 2007;3:301-16.

5. Chou CS, Chen CY, Lin SH, Lu WH, Wu P. Preparation of $\mathrm{TiO}_{2} /$ bamboo-charcoal-powder composite particles and their applications in dye-sensitized solar cells. Adv Powder Tech 2015;26:711-7.

6. Guzman M, Dille J, Godet S. Synthesis and antibacterial activity of silver nanoparticles against Gram-positive and Gram-negative bacteria. Nanomed Nanotech Bio Med 2012;8:37-45

7. Bian X, Lu X, Jin E, Kong L, Zhang W, Wang C, et al. Fabrication of PT/polypyrrole hybrid hollow microspheres and their application in electrochemical biosensing towards hydrogen peroxide. Talanta 2010;81:813-8.

8. Banerjee A, Theron R, Scott RW. Redispersion of sinthered nanoparticles catalysts in tetraalkylphosphonium ionic liquid. J Mol Catal Chem 2014;393:105-11.

9. Peng S, McMahon JM, Schatz GC, Gray SK, Sun Y. Reversing the sizedependence of surface plasmon resonances. Proc Natl Acad Sci U S A 2010;107:14530-4.

10. Sabatini CA, Pereira RV, Gehlen MH. Fluorescence modulation of acridine and coumarin dyes by silver nanoparticles. J Fluores 2007; 17:377-82.

11. Setua P, Ghatak C, Rao VG, Das SK, Sarkar N. Dynamics of solvation and rotational relaxation of coumarin 480 in pure aqueous-AOT reverse micelle and reverse micelle containing different-sized silver nanoparticles inside its core: A comparative study. J Phys Chem 2012;116:3704-12.

12. Solomon MM, Umoren SA. In-situ preparation, characterization and anticorrosion property of polypropylene glycol/silver nanoparticles composite for mild steel corrosion in acid solution. J Colloid Interface Sci 2016;462:29-41

13. Nia PM, Meng WP, Alias Y. Hydrogen peroxide sensor: Uniformly decorated silver nanoparticles on polypyrrole for wide detection range. App Surf Sci 2015;357:1565-72.

14. Deshpande R, Ravishankar B, Sharanbasava G, Mahesh DB, Harsoor V, Yalagatti MS, et al. Anti-cancer studies of noble metal nanoparticles synthesized using different plant extracts. Cancer Nanotech 2011;2:57-65.

15. Ragunathan D, Basavaraj S, Sawle B, Manjunath SY. Biosynthesis of stable polyshaped gold nanoparticles from microwave-exposed aqueous extracellular anti-malignant guava (Psidium guajava) leaf extract. Nanobiotechnology 2009;5:34-41.

16. Teixeira RS, Camparoto ML, Mantovani MS, Vicentini VE. Assesment of two medicinal plants, Psidium guajava L. and Achillea millefolium. $\mathrm{L}$ in vitro and in vivo assays. Genet Mol Biol 2003;26:234-9.

17. Ojewolw JE. Anti inflamatory and analgesic effects of Psidium guajava Linn. (Myrtacaea) leaf aqueous extract in rats and mice. Met Fin Exp Clini Pharma 2006;28:441-6.

18. Bang LS, Ryong PH. Anticancer activity of guava (Psidium guajava 1.) branch extract against HT-29 human colon cancer cells. J Med Plant 
Res 2010;4:891-6.

19. Ramadhania ZM, Insanu M, Gunarti NS, Wirasutisna KR, Sukrasno S, Hartati R. Antioxidant activity from ten species of Myrtaceae. Asian J Pharm Clin Res 2017;5-7.

20. Parashar UK, Kumar V, Bera T, Saxena PS, Nath G, Srivastava SK, et al. Study of mechanism of enhanced antibacterial activity by green synthesis of silver nanoparticles. Nanotechnology 2011;22:415104.

21. Xiao-Long Y, Kun-Lung H, Ji-Kai L. Guajadial: An unusual metroterpenoid from guava leaves Psidium guajava. Org Lett 2007:9:5135-8.

22. Gutierrez RM, Mitchell S, Solis RV. Psidium guajava: A review of its traditional uses, phytochemistry and pharmacology. J Ethnopharma 2008;117:1-27.

23. Deshpande R, Bedre DM, Basavaraja S, Balaji SD, Manjunath SY, Venkataraman A, et al. Microwave-assisted rapid extracellular synthesis of stable bio-functionalized silver nanoparticles from guava (Psidium guajava) leaf extract. J Nanopart Res 2011;13:2021-8.

24. Shirur DS, Adhikari R, Sanjay ST, Sajjekhan S. A review on the medicinal plant Psidium guajava. J Drug Deliv Ther 2013;3:162-8.

25. Rajamani R, Kuppusamy S, Shamugavadivu M. Green synthesis of silver nanoparticles using areca nut extract for enhanced antibacterial activity. J Green Sci Technol 2013;1:102-6.

26. Sharmila C, Vinuppriya R, Chandran S, Chemmanda J, Chandarsheka B. Biosynthesis of PVA encapsulated silver nanoparticles. J App Res Tech 2016;14:319-24.

27. MubarakAli D, Thajuddin N, Jeganathan K, Gunasekaran M. Plant extract mediated synthesis of silver and gold nanoparticles and its antibacterial activity against clinically isolated pathogens. Colloids Surf B Biointerfaces 2011;85:360-5.

28. Tanakae T, Ishida N, Ishimatsu M, Nonaka G, Nishioka I. Tannins and related compounds CXVI. Tannins, guajavins psidinins and psiguavin from the bank of Psidium guajava leaf. Chem Pharm Bull
1992;40:2092-8

29. Jayeeta B, Neeraj ST, Kirar S, Banerjee UC. Bioinspired nanotheranostic agents: Synthesis, surface functionalization, and antioxidant potential. ACS Biomater Sci Eng 2015;1:382-92.

30. Bankura KP, Maity D, Mollick MM, Mondal D, Bhowmick B, Bain MK, et al. Synthesis, characterization and antimicrobial activity of dextran stabilized silver nanoparticles in aqueous medium. Carbohydr Polym 2012;89:1159-65.

31. Ragunath A, Nisha P, Kumuthakalavalli K. Mycosynthesis of silver nanoparticles: Characterization, antioxidant and anti-inflammatory activity from Pleurotus florida (Mont) singer: A macro fungi. Asian J Pharm Clin Res 2017;10:186-91.

32. Coseri S, Spatareanu A, Sacarescu L, Rimbu C, Suteu D, Spirk S, et al. Green synthesis of the silver nanoparticles mediated by pullulan and 6-carboxypullulan. Carbohydr Polym 2015;116:9-17.

33. Kirthika P, Dheeba B, Sivakumar R, Abdulla SS. Plant mediated synthesis and characterization of silver nanoparticles. Int $\mathrm{J}$ Pharm Pharm Sci 2014;6:304-10.

34. Mittal AK, Bhaumik J, Kumar S, Banerjee UC. Biosynthesis of silver nanoparticles: Elucidation of prospective mechanism and therapeutic potential. J Coll Int Sci 2014;415:39-47.

35. Sondi I, Salopek-Sondi B. Silver nanoparticles as antimicrobial agent: A case study on $E$. Coli as a model for gram-negative bacteria. J Colloid Interface Sci 2004;275:177-82.

36. Murugesan S, Bhuvaneshwari S, Sivamurugan V. Green synthesis characterization of silver nanoparticles of marine red alga spyridia fusiformis and their antibacterial activity. Int $\mathrm{J}$ Pharm Pharm Sci 2017;5:192-7

37. Sengodan R, Dinesh KP, Ranjithkumar R, Sagadevan P, Sathish S, Chandar Shekar B. Synthesis, characterization and remedial aspect of BaTiO3 nanoparticles against bacteria. Nanomed Nanobiotech $2014 ; 1: 1-5$ 